\title{
Efecto antinociceptivo de estriol y Glicina max I. (soya) en ratas ovariectomizadas
}

Antinociceptive effect of estriol and Glicina max I. (soya) in ovariectomized rats

\author{
Reyna Ángeles-Palacios ${ }^{1}$, Leidy Bacalla ${ }^{1}$, Karen Campos ${ }^{1}$, Yuly Cusihuamán", \\ Milagros Osorio ${ }^{1}$, Daisy Flores ${ }^{2}$ \\ Estudiantes de la EAP Obstetricia, Facultad de Medicina, UNMSM. \\ ${ }^{2}$ Profesor de Farmacologia, Facultad de Medicina, UNMSM
}

\begin{abstract}
Resumen
Objetivo: Demostrar el efecto protector del estriol y Glicina max L. (soya) frente al dolor nociceptivo, en ratas ovariectomizadas. Diseño: Estudio experimental. Lugar: Bioterio de la Facultad de Medicina, Universidad Nacional Mayor de San Marcos. Material biológico: Ratas hembras albinas Holtzman de 2,5 a 3 meses de edad. Métodos: Las 29 ratas fueron agrupadas aleatoriamente: grupo 1 ( $n=8)$, no ovariectomizadas, tratadas con solución salina $(0,1 \mathrm{~mL} / \mathrm{kg})$; grupo $2(\mathrm{n}=7)$, ovariectomizadas y tratadas con solución salina $(0,1 \mathrm{~mL})$ $\mathrm{kg})$; grupo $3(\mathrm{n}=7)$, ovariectomizadas y tratadas con estriol $(0,2 \mathrm{mg} / \mathrm{kg})$; grupo $4(\mathrm{n}=7)$, ovariectomizadas y tratadas con Glycine max $\mathrm{L}$. $(60 \mathrm{mg} / \mathrm{kg})$. Se realizó una prueba basal de dolor térmico en el hornillo a todos los animales, asi como tres días después del tratamiento farmacológico. Principales medidas de resultados: Se usó el porcentaje de eficiencia antinociceptiva máxima (\%EAM). Resultados: Se encontró que el \%EAM fue mayor en el grupo de ratas no ovariectomizadas $(1,6 \%)$ que en el grupo de las ovariectomizadas $(0,8 \%)$. Por otro lado, el \%EAM en ratas ovariectomizadas con solución salina $(0,8 \%)$ fue menor al compararlo con el grupo de las tratadas con estriol (5,8\%); asi también, el \%EAM del grupo de ovariectomizadas tratadas con Glicina max L. (4,2\%) fue superior al grupo de ovariectomizadas tratadas con solución salina $(0,8 \%)$, pero esta no fue superior a las del grupo tratadas con estriol. Conclusiones: $E$ estriol y la Glicina max L. ejercieron un papel protector frente al dolor nociceptivo térmico en ratas ovariectomizadas.

Palabras clave: Dolor, estriol, Glicina max L, ratas ovariectomizadas, hornillo.

\section{Abstract}

Objective: To determine the protective effect of estriol and Glycine max L. (soybean) compared to nociceptive pain in ovariectomized rats. Design: Experimental study. Setting: Faculty of Medicine bioterium, Universidad Nacional Mayor de San Marcos, Lima, Peru. Biological material: Holtzman albino female rats 2,5 to 3 months of age. Methods: The 29 rats were grouped randomly: group $1(n=8)$ non ovariectomized rats treated with saline $(0.1 \mathrm{~mL} / \mathrm{kg})$; group $2(n=7)$ ovariectomized rats treated with saline $(0.1 \mathrm{~mL} / \mathrm{kg}) ;$ group $3(n=$ 7) ovariectomized rats treated with estriol $(0.2 \mathrm{mg} / \mathrm{kg})$; group $4(\mathrm{n}=7)$ ovariectomized rats treated with Glycine max $\mathrm{L}$. $(60 \mathrm{mg} / \mathrm{kg})$. We determined baseline hotplate thermal pain as well as three days after treatment. Main outcome measures: Antinociceptive maximum efficacy (\% AME). Results: We found that \% EAM was higher in the non ovariectomized rats group (1.6\%) than in the ovariectomized group $(0.8 \%)$. Furthermore, the percentage of EAM in ovariectomized rats treated with saline $(0.8 \%)$ compared with the group treated with estriol (5.8\%) and also the percentage of EAM in the Glycine max L. group (4.2\%) was higher than in the ovariectomized group treated with saline $(0.8 \%)$ but not higher than the group treated with estriol. Conclusions: Estriol and Glycine max L. have protective role against thermal nociceptive pain in ovariectomized rats.
\end{abstract}

Key words: Pain, estriol, Glycine max L., ovariectomized rats, hot plate.

An Fac med. 2011;72(1):17-22

\section{INTRODUCCIÓN}

El dolor, aunque indeseable, representa una estrategia adaptativa que permite protegernos de las agresiones del medio externo. Sin la presencia de estas respuestas ante un estímulo doloroso, tendríamos una desventaja de supervivir. Sin embargo, en algunas circunstancias el dolor pasa de ser una sensación benéfica para el organismo y se convierte en una patología que debe ser suprimida para permitirle al organismo sobrevivir.
El estudio del dolor implica una amplia gama de conocimientos. La Asociación Internacional para el Estudio del Dolor (IASP) define al dolor como "una experiencia emocional y sensorial desagradable asociada a un daño tisular actual, real o potencial, o descrito en términos de dicho daño".

Hoy en día, entendemos el dolor como la integración de tres componentes: a) el sensitivo, que hace referencia al impulso desencadenado desde los receptores periféricos de dolor; b) el componente cognitivo se relaciona con nuestro aprendizaje cultural respecto al dolor y con las conductas que tomamos como reacción a este; integra factores tales como el entorno social y cultural, aprendizajes previos, otros; c) el tercer componente es el emotivo-afectivo, que hace referencia a nuestras emociones frente a un impulso doloroso y la manera como pueden influir en la interpretación del mismo ${ }^{(1)}$.

El dolor puede ser dividido en dos categorías: nociceptivo y neuropático. 
El dolor nociceptivo es causado por la estimulación de un sistema nervioso intacto que funciona normalmente. Es beneficioso para el organismo, ya que invoca acciones de protección y defensa para evitar mayor daño y para ayudar en la reparación tisular y regeneración. Por la diferencia en el patrón de inervación, el dolor nociceptivo puede ser clasificado como somático, es decir, producido por la activación de los nociceptores de la piel, hueso y partes blandas; por ello es un dolor sordo, continuo y bien localizado en las áreas superficiales muy inervadas, presentando una respuesta rápida frente a estímulos de tipo mecánico, térmico y visceral, ocasionado por la activación de nociceptores por infiltración, compresión, distensión, tracción o isquemia de vísceras pélvicas, abdominales o torácicas; por ello, se trata de un dolor localizado pobremente, descrito a menudo como profundo y opresivo.

En contraste, el dolor neuropático es causado por un sistema nervioso con función alterada. La injuria del sistema nervioso y la patología causal pueden encontrarse a cualquier nivel del neuroaxis ${ }^{(2)}$.

El conocimiento de estos dolores nos ha permitido llegar a un mejor manejo de los mismos, por medio de múltiples tratamientos. Si bien se tiene toda una gama de medicamentos que tratan el dolor, no todos los dolores han podido ser controlados: es el caso del dolor lumbar, neuropatía trigeminal y otros. Existe la necesidad de buscar nuevos conocimientos que pudieran contribuir al alivio para estos tipos de dolor. Hay estudios que relacionan los estrógenos con la disminución del dolor, aunque existen controversias respecto a esta relación. En ausencia de información definitiva, decidimos realizar este trabajo experimental, con el objetivo de demostrar el efecto antinociceptivo del estriol en ratas ovariectomizadas, así como comparar la potencia antinociceptiva del estriol frente a un fitoestrógeno -Glicina max (soya)-, ya que las semillas de soya contienen importantes sustancias nutritivas -albúminas, lípi- dos, lecitinas, hidratos de carbono y vitaminas-que le dan la capacidad de tener un efecto protector ante el dolor ${ }^{(3)}$. Empleamos modelos animales experimentales (ratas ovariectomizadas) para observar los efectos de los estrógenos sobre el dolor. Nos planteamos como objetivo demostrar el efecto antinociceptivo del estriol y Glicina max L. en ratas ovariectomizadas.

\section{MÉTODOS}

El diseño del trabajo corresponde a un estudio experimental, en el que se empleó una muestra de 29 ratas hembras albinas Holtzman de 2,5 a 3 meses de edad, obtenidas del bioterio de la Universidad Nacional Agraria La Molina (UNALM). Posteriormente, fueron mantenidas en el bioterio de la Facultad de Medicina de la Universidad Nacional Mayor de San Marcos (UNMSM), donde tuvieron una semana de adaptación.

Para la demostración del trabajo experimental, se realizó la ovariectomía, para evaluar la influencia del reemplazo hormonal sobre las respuestas al dolor y la analgesia en este modelo animal ${ }^{(4)}$.

Para la ovariectomía se requirió del anestésico ketamina, administrada por vía intraperitoneal, a dosis de $75 \mathrm{mg} / \mathrm{kg}$. Después de la cirugía, las ratas tuvieron una semana de recuperación en un ambiente con una temperatura adecuada, proporcionada por una estufa.

Se agrupó a los animales de manera aleatoria, siendo ocho ratas para el grupo de las no ovariectomizadas y 21 ratas para el grupo de las ovariectomizadas. Posteriormente, se procedió a la realización del tratamiento de reemplazo hormonal (TRH). A partir del grupo de ratas ovariectomizadas, se formó 3 subgrupos:

- Grupo 1: $(\mathrm{n}=8)$ ratas no ovariectomizadas.

- Grupo 2: $(n=7)$ ratas ovariectomizadas tratadas con solución salina 1 $\mathrm{mL} / 100 \mathrm{~g}$.
- Grupo 3: $(n=7)$ ratas ovariectomizadas que recibieron estriol intragástrico a dosis de $0,2 \mathrm{mg} / \mathrm{kg}$.

- Grupo 4: $(n=7)$ ratas ovariectomizadas que recibieron Glicina max L. (soya) a dosis de $60 \mathrm{mg} / \mathrm{kg}$.

Después de recibir el tratamiento por tres días, los animales fueron colocados en el hornillo (hot plate), equipo que consiste en una placa conectada a una resistencia termostática, de manera que se obtiene la temperatura deseada; además, posee un cilindro acrílico transparente para observar la respuesta dolorosa y evitar la huída del animal.

Los animales fueron evaluados en el hornillo a $55^{\circ} \mathrm{C}$, registrándose el periodo de latencia de la reacción nociceptiva. Este valor viene determinado por los segundos que tarda la rata en experimentar un signo de dolor (reflejo de lamer las patas delanteras).

La evaluación del efecto antinociceptivo se hizo por medio del porcentaje del efecto antinociceptivo máximo (\%EAM) posible, mediante la siguiente fórmula:

$\% \mathrm{EAM}=(\mathrm{PL}-\mathrm{PL} \underset{\text { post to }}{\mathrm{pre} \mathrm{tto}}) \times 100 /(\mathrm{TC}-\mathrm{PL} \underset{\text { post }}{)}$

donde $\mathrm{PL}_{\text {post to }}$ es el periodo de latencia postratamiento y $\mathrm{PL}_{\text {pre too }}$, periodo de latencia antes del tratamiento, y TC es el tiempo de corte en el cual la prueba será terminada en ausencia de respuesta (30 segundos).

Con relación a las consideraciones éticas, el procedimiento aplicado a los animales para inducir la anestesia fue realizado con el mayor cuidado, ya que alguna falla en el procedimiento pudo haber tenido consecuencias no deseables. Como se mencionó, se utilizó ketamina, fármaco usado frecuentemente en veterinaria para intervenciones preoperatorias. Por otro lado, la eutanasia aplicada a los animales permitió una rápida pérdida de conciencia sin dolor o miedo, seguido por un alivio progresivo, hasta la muerte del animal.

Para efectos del experimento, se eligió el método de eutanasia inducido 
Tabla 1. Promedio y desviación típica de periodo de latencia basal, según grupos de tratamiento.

\begin{tabular}{|c|c|c|c|}
\multicolumn{1}{c|}{} & N & Media (s) & Desviación típica \\
\hline Grupo 1 & 8 & 6,2 & 1,0 \\
Grupo 2 & 7 & 4,3 & 0,6 \\
Grupo 3 & 7 & 5,0 & 0,9 \\
Grupo 4 & 7 & 4,3 & 0,5 \\
\hline Total & 29 & & \\
\hline
\end{tabular}

Grupo 1: $(n=8)$ ratas no ovariectomizadas.

Grupo 2: $(\mathrm{n}=7)$ ratas ovariectomizadas tratadas con solución salina $1 \mathrm{~mL} / 100 \mathrm{~g}$.

Grupo 3: ( $\mathrm{n}=7$ ) ratas ovariectomizadas que recibieron estriol intragástrico, a dosis de $0,2 \mathrm{mg} / \mathrm{kg}$.

Grupo 4: $(n=7)$ ratas ovariectomizadas que recibieron Glicina max L.(soya), a dosis de $60 \mathrm{mg} / \mathrm{kg}$.

Tabla 2. Promedio y desviación típica de periodo de latencia post tratamiento en los grupos experimentales.

\begin{tabular}{|c|c|c|c|}
\multicolumn{1}{c|}{} & N & Media (s) & Desviación típica \\
\hline Grupo 1 & 8 & 6,4 & 1,3 \\
Grupo 2 & 7 & 4,5 & 0,7 \\
Grupo 3 & 7 & 5,5 & 1,1 \\
Grupo 4 & 7 & 6,1 & 1,2 \\
\hline Total & 29 & & \\
\hline
\end{tabular}

Tabla 3. Promedio y desviación típica de \% EAM en grupos, según tratamiento.

\begin{tabular}{|c|c|c|c|}
\multicolumn{1}{c|}{} & N & Media (s) & Desviación típica \\
\hline Grupo 1 & 8 & 1,6 & 2,8 \\
Grupo 2 & 7 & 0,8 & 2,2 \\
Grupo 3 & 7 & 5,8 & 6,5 \\
Grupo 4 & 7 & 4,2 & 5,5 \\
\hline Total & 29 & & \\
\hline
\end{tabular}

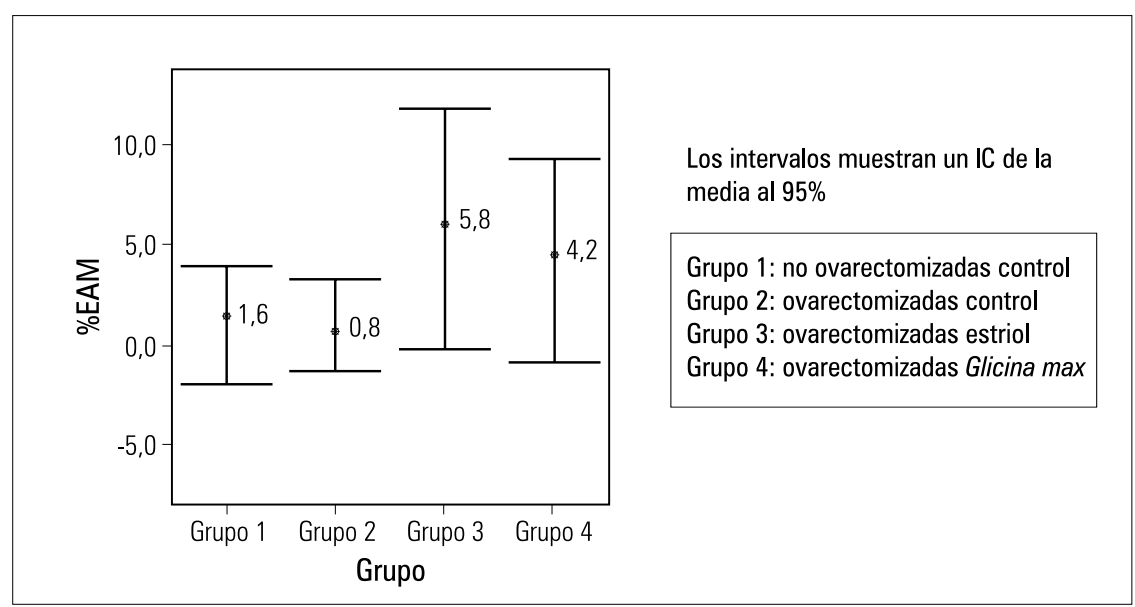

Figura 1. \% EAM (eficiencia antinociceptiva máxima) en ratas, según grupos de tratamiento. por anestésicos inhalatorios, considerado un método aceptable para roedores por el Report of the AVMA Panel on Euthanasia (1993) y el Euthanasia of Experimental Animals, de la Unión Europea $(1995)^{(5)}$.

Se empleó éter etílico, embebido en una torunda de algodón y colocado en el interior de una campana de vidrio junto con el animal, y se esperó la muerte del mismo.

\section{RESULTADOS}

Los resultados son mostrados en las siguientes tablas y gráficos.

En el tabla 1 se observa que el grupo 1 presentó mayor periodo de latencia basal (6,2 s) frente al dolor, mientras que los grupos 2 y 4 coincidieron con el menor periodo de latencia (4,3 s).

En la tabla 2 se observa que el grupo de ratas no ovariectomizadas presentó mayor periodo de latencia $(6,4 \mathrm{~s})$, sin considerar los datos basales, mientras que el grupo de las ovariectomizadas tratadas con solución salina presentó el menor periodo de latencia (4,5 s).

En la tabla 3 se observa el \%EAM de los cuatro grupos, donde el grupo de ratas ovariectomizadas tratadas con estriol presentó mayor efecto antinociceptivo (5,8 s), mientras que el grupo de las ovariectomizadas tratadas con solución salina obtuvo el menor efecto protector contra el dolor $(0,8 \mathrm{~s})$.

Analizadas las tablas estadísticas, se procedió a elaborar la figura 1 .

En esta figura se evidencia el mayor efecto protector frente al dolor nociceptivo en el grupo de ratas no ovariectomizadas control $(1,6 \%)$, con respecto al grupo de ratas ovariectomizadas control $(0,8 \%)$. Dentro del grupo de las ratas ovariectomizadas, encontramos que al comparar el efecto antinociceptivo del grupo tratado con estriol y Glicina max, el primero fue mayor. Por otro lado, se puede decir que tanto el grupo de estriol como el Glicina max L. proveen de mayor protección frente al dolor nociceptivo, al compararlos con el grupo de ovariectomizadas control. 


\section{DISCUSIÓN}

En la experiencia de dolor somático en ratas ovariectomizadas y no ovariectomizadas, pre y postratamiento, hallamos los siguientes porcentajes de efectos antinociceptivo máximo (\%EAM): el grupo conformado por ratas no ovariectomizadas presentó 1,6\% EAM, el grupo de ratas ovariectomizadas tratadas con suero fisiológico tuvo 0,8\% EAM, el grupo de ratas ovariectomizadas con tratamiento de estriol presentó 5,8\% EAM y el grupo de ratas ovariectomizadas tratadas con Glicina max L.(soya), 4,2\% EAM.

Encontramos que el estrógeno endógeno tuvo un efecto protector frente al dolor somático provocado, ya que el grupo de ratas no ovariectomizadas presentó un mayor \%EAM, en comparación con el grupo de ratas ovariectomizadas tratadas con solución salina, quienes no contaban con la principal fuente de estrógeno endógeno. Estos resultados coinciden con otros trabajos presentados anteriormente. Uno de ellos es el estudio publicado por Mannino y col. ${ }^{(6)}$, donde se demostró el efecto protector del $17 \beta$ estradiol frente al dolor visceral, a concentraciones de 5 a $40 \%$, utilizando la prueba de formalina, presentándose el efecto antinociceptivo máximo al 20\% de concentración de $17 \beta$ estradiol.

Por otro lado, el grupo de ratas ovariectomizadas tratadas con estriol presentó mayor \%EAM en comparación con el grupo de ratas ovariectomizadas que recibieron solución salina. Podemos afirmar que el estriol tendría un efecto antinociceptivo (protección frente al dolor visceral como somático) en ratas. Este resultado contribuye al conjunto de estudios que prueban que hay una relación entre los estrógenos y la sensibilidad al dolor. Aunque no se conoce bien los mecanismos subyacentes que puedan revelar dicha relación, una posible explicación se basa en que los estrógenos tienen acción sobre ciertas funciones del cerebro, como el desarrollo neuronal y la supervivencia de las células nerviosas, a través de sus receptores estrogénicos llamados ERalfa y ER-beta ${ }^{(7)}$, que se encuentran localizados en las áreas del tallo cerebral (núcleo periventricular y en el núcleo trigéminal) y la médula espinal (en las láminas I y II $)^{(8)}$.

Ciertos estudios apoyan la idea de que los estrógenos aumentan la sensibilidad al dolor (disminuyen el umbral del dolor). Así también, hay otros estudios que sostienen que la contribución de los estrógenos ayuda a la disminución de la sensibilidad al dolor. La primera idea planteada se apoya en que la administración de estrógenos en las mujeres puede aumentar la incidencia del dolor, debido a las acciones que los estrógenos ejercen a nivel periférico y central, como por ejemplo, aumentan los factores de crecimiento nervioso (NGF) en los ganglios de la raíz posterior. Otra acción es que excitan rápidamente a las neuronas que se encuentran en la corteza cerebral, en el cerebelo y el hipocampo. Estos efectos pueden aumentar la nocicepción ${ }^{(9)}$. La segunda idea planteada se apoya en que los receptores ER-beta desempeñan un papel importante en el desarrollo de las fibras nerviosas de la médula espinal, que son las que transportan la información al cerebro. Entre otras cosas, estos nervios determinan la sensibilidad al dolor y la respuesta a las sensaciones en general. Todo ello nos lleva a pensar que las sustancias que estimulan el receptor ER-beta pueden aliviar el dolor. A este mecanismo se añade otra posible explicación, que se encuentra relacionada con el hecho de que los estrógenos regulan el control transcripcional de la síntesis de opioides y de los receptores opioides delta $(\delta)$ y kappa $(\kappa)$ en la lámina II de la médula espinal ${ }^{(10)}$.

La administración de estrógeno en las mujeres aumenta la unión en el cerebro de opioide-receptor opioideo mu $(\mu)$ inducido por el dolor, lo que sugiere que el estrógeno mejora el funcionamiento del sistema opioide endógeno, provocando así un aumento del umbral del dolor.

Otros estudios realizados en mujeres posmenopáusicas han sustentado que la terapia de reemplazo hormonal con estrógeno es muy eficaz en la disminución de la sensibilidad al dolor causado por problemas articulares (dolores de espalda y artritis) ${ }^{(11)}$.

Además, en nuestra experiencia hemos encontrado que el fitoestrógeno Glicina max L. (soya) tiene también un efecto protector frente al dolor somático, en un \%EAM de 4,2\%, mayor en comparación con el grupo de ratas ovariectomizadas tratadas con suero fisiológico. Nuestro resultado concuerda con lo demostrado en estudios anteriores, como la investigación de Shir y col. ${ }^{(12)}$ en ratas macho; este estudio consistió en ligarles el nervio ciático para posteriormente causarles dolor neuropático. Ellos trabajaron con cuatro grupos de ratas; tres de estos recibieron dieta de Glicina max L. (soya) en diferentes concentraciones y un último grupo recibió dieta carente de Glicina max L. Para la prueba de dolor, se estimuló con un cabello la zona plantar de la pata que tenía el nervio afectado, concluyendo que el grupo de ratas que tenía un nivel medio de Glicina max L. en sangre presentó una reducción tanto de alodinia táctil como de hiperalgesia mecánica, en comparación con el grupo de ratas cuya dieta carecía de soya, el que presentó un aumento de alodinia táctil.

Una explicación posible para el efecto protector de Glicina max L. es que las concentraciones de fitoestrógeno a nivel central pueden aumentar el factor neurotrófico e inhibir la tirosina quinasa, que desempeñan un papel importante en la transmisión de la sensación dolorosa ${ }^{(13)}$.

Finalmente, hemos encontrado que el grupo de ratas ovariectomizadas tratadas con estriol presentó un \%EAM ligeramente mayor, en comparación con el grupo de ratas tratadas con Glicina max.

En la actualidad, la medicina y la tecnología se encuentran en una constante lucha por lograr el manejo del dolor $y$, aunque ha habido un gran avance, aún existen limitaciones en este campo. Es por ello que consideramos de suma importancia los resultados hallados en 
este trabajo experimental, pues pretendemos dar inicio a futuros estudios que permitan encontrar nuevas vías de nocicepción, a través de receptores de estrógenos. Así también, el saber que en la gestante el uso de fármacos analgésicos es en su mayoría restringido, pretendemos que este trabajo de investigación sea un punto de partida para posteriores investigaciones que busquen la elaboración de nuevos fármacos, cuya estructura sea equivalente o similar a los estrógenos, pero con efectos inocuos en la gestación.

Podemos concluir que tanto el estriol como la Glicina max (soya) presentaron efecto antinociceptivo frente al dolor térmico inducido en ratas ovariectomizadas, aunque dicho efecto fue menor con la soya comparada con el estriol; esta diferencia no fue significativa.En la experiencia de dolor somático en ratas ovariectomizadas y no ovariectomizadas, pre y postratamiento, hallamos los siguientes porcentajes de efectos antinociceptivo máximo (\%EAM): el grupo conformado por ratas no ovariectomizadas presentó 1,6\% EAM, el grupo de ratas ovariectomizadas tratadas con suero fisiológico tuvo 0,8\% EAM, el grupo de ratas ovariectomizadas con tratamiento de estriol presentó 5,8\% EAM y el grupo de ratas ovariectomizadas tratadas con Glicina max L.(soya), 4,2\% EAM.

Encontramos que el estrógeno endógeno tuvo un efecto protector frente al dolor somático provocado, ya que el grupo de ratas no ovariectomizadas presentó un mayor \%EAM, en comparación con el grupo de ratas ovariectomizadas tratadas con solución salina, quienes no contaban con la principal fuente de estrógeno endógeno. Estos resultados coinciden con otros trabajos presentados anteriormente. Uno de ellos es el estudio publicado por Mannino y col. (6), donde se demostró el efecto protector del $17 \beta$ estradiol frente al dolor visceral, a concentraciones de 5 a $40 \%$, utilizando la prueba de formalina, presentándose el efecto antinociceptivo máximo al $20 \%$ de concentración de $17 \beta$ estradiol.
Por otro lado, el grupo de ratas ovariectomizadas tratadas con estriol presentó mayor \%EAM en comparación con el grupo de ratas ovariectomizadas que recibieron solución salina. Podemos afirmar que el estriol tendría un efecto antinociceptivo (protección frente al dolor visceral como somático) en ratas. Este resultado contribuye al conjunto de estudios que prueban que hay una relación entre los estrógenos y la sensibilidad al dolor. Aunque no se conoce bien los mecanismos subyacentes que puedan revelar dicha relación, una posible explicación se basa en que los estrógenos tienen acción sobre ciertas funciones del cerebro, como el desarrollo neuronal y la supervivencia de las células nerviosas, a través de sus receptores estrogénicos llamados ERalfa y ER-beta ${ }^{(7)}$, que se encuentran localizados en las áreas del tallo cerebral (núcleo periventricular y en el núcleo trigéminal) y la médula espinal (en las láminas I y II ) ${ }^{(8)}$.

Ciertos estudios apoyan la idea de que los estrógenos aumentan la sensibilidad al dolor (disminuyen el umbral del dolor). Así también, hay otros estudios que sostienen que la contribución de los estrógenos ayuda a la disminución de la sensibilidad al dolor. La primera idea planteada se apoya en que la administración de estrógenos en las mujeres puede aumentar la incidencia del dolor, debido a las acciones que los estrógenos ejercen a nivel periférico y central, como por ejemplo, aumentan los factores de crecimiento nervioso (NGF) en los ganglios de la raíz posterior. Otra acción es que excitan rápidamente a las neuronas que se encuentran en la corteza cerebral, en el cerebelo y el hipocampo. Estos efectos pueden aumentar la nocicepción ${ }^{(9)}$. La segunda idea planteada se apoya en que los receptores ER-beta desempeñan un papel importante en el desarrollo de las fibras nerviosas de la médula espinal, que son las que transportan la información al cerebro. Entre otras cosas, estos nervios determinan la sensibilidad al dolor y la respuesta a las sensaciones en general. Todo ello nos lleva a pensar que las sustancias que estimulan el receptor ER-beta pueden aliviar el dolor. A este mecanismo se añade otra posible explicación, que se encuentra relacionada con el hecho de que los estrógenos regulan el control transcripcional de la síntesis de opioides y de los receptores opioides delta $(\delta)$ y kappa $(\kappa)$ en la lámina II de la médula espinal ${ }^{(10)}$.

La administración de estrógeno en las mujeres aumenta la unión en el cerebro de opioide-receptor opioideo mu $(\mu)$ inducido por el dolor, lo que sugiere que el estrógeno mejora el funcionamiento del sistema opioide endógeno, provocando así un aumento del umbral del dolor.

Otros estudios realizados en mujeres posmenopáusicas han sustentado que la terapia de reemplazo hormonal con estrógeno es muy eficaz en la disminución de la sensibilidad al dolor causado por problemas articulares (dolores de espalda y artritis) ${ }^{(11)}$.

Además, en nuestra experiencia hemos encontrado que el fitoestrógeno Glicina max L. (soya) tiene también un efecto protector frente al dolor somático, en un \%EAM de 4,2\%, mayor en comparación con el grupo de ratas ovariectomizadas tratadas con suero fisiológico. Nuestro resultado concuerda con lo demostrado en estudios anteriores, como la investigación de Shir y col. ${ }^{(12)}$ en ratas macho; este estudio consistió en ligarles el nervio ciático para posteriormente causarles dolor neuropático. Ellos trabajaron con cuatro grupos de ratas; tres de estos recibieron dieta de Glicina max L. (soya) en diferentes concentraciones y un último grupo recibió dieta carente de Glicina max L. Para la prueba de dolor, se estimuló con un cabello la zona plantar de la pata que tenía el nervio afectado, concluyendo que el grupo de ratas que tenía un nivel medio de Glicina max L. en sangre presentó una reducción tanto de alodinia táctil como de hiperalgesia mecánica, en comparación con el grupo de ratas cuya dieta carecía de soya, el que presentó un aumento de alodinia táctil. 
Una explicación posible para el efecto protector de Glicina max L. es que las concentraciones de fitoestrógeno a nivel central pueden aumentar el factor neurotrófico e inhibir la tirosina quinasa, que desempeñan un papel importante en la transmisión de la sensación dolorosa ${ }^{(13)}$.

Finalmente, hemos encontrado que el grupo de ratas ovariectomizadas tratadas con estriol presentó un \%EAM ligeramente mayor, en comparación con el grupo de ratas tratadas con Glicina $\max$.

En la actualidad, la medicina y la tecnología se encuentran en una constante lucha por lograr el manejo del dolor $y$, aunque ha habido un gran avance, aún existen limitaciones en este campo. Es por ello que consideramos de suma importancia los resultados hallados en este trabajo experimental, pues pretendemos dar inicio a futuros estudios que permitan encontrar nuevas vías de nocicepción, a través de receptores de estrógenos. Así también, el saber que en la gestante el uso de fármacos analgésicos es en su mayoría restringido, pretendemos que este trabajo de investigación sea un punto de partida para posteriores investigaciones que busquen la elaboración de nuevos fármacos, cuya estructura sea equivalente o similar a los estrógenos, pero con efectos inocuos en la gestación.

Podemos concluir que tanto el estriol como la Glicina max (soya) presentaron efecto antinociceptivo frente al dolor térmico inducido en ratas ovariectomizadas, aunque dicho efecto fue menor con la soya comparada con el estriol; esta diferencia no fue significativa.

\section{REFERENCIAS BIBLIOGRÁFICAS}

1. Dagnino J. Definiciones y clasificaciones de dolor. Bol Esc Med Univ Catol Chile. 1994;23:148-51.

2. Miller R, Abram SE. Atlas of Anesthesia: Pain management. 1a. ed. Philadelphia: WB Saunders, 1999.

3. Abbott FV, Young SN. The effect of tryptophan supplementation on autonomy induced by nerve lesions in rats. Pharmacol Biochem Behav. 1991;40:301-4

4. Fillingim RB, Ness TJ. Sex-related hormonal influences on pain and analgesic responses. Neurosci Biobehav Rev. 2000;24:485-501.

5. Close B, Banister K, Baumans V., Bernoth E, Bromage N, Bunyan J, Erhardt W y col. Recomendaciones para la eutanasia de los animales de experimentación Lab Animal. 1997;31;1-32.

6. Mannino CA, South SM, Quinones-Jenab V. Estradiol replacement in ovariectomized rats is antihyperalgesic in the formalin test. J Pain. 2007;8(4):334-42.

7. International Association for the Study of Pain. Diferencias de Dolor entre los Hombres y las Mujeres. Septiembre 2007 Disponible en: http://
www.iasp-pain.org/AM/Template.cfm? Acceso: 15 de julio del 2007.

8. Taylor BK, Akana SF, Peterson MA, Dallman MF, Basbaum Al. Pituitary-adrenocortical responses to persistent noxious stimuli in the awake rat: endogenous corticosterone does not reduce nociception in the formalin test. Endocrinol. 1998;139:2407-13.

9. Gaumond I, Arsenault P, Marchand S: Specificity of female and male sex hormones on excitatory and inhibitory phases of formalin - induced nociceptive responses. Brain Res. 2002;1052:139-45.

10. Fan X, Kim HJ, Warner M, Gustafsson JA. Estrogen receptor beta is essential for sprouting of nociceptive primary afferents and for morphogenesis and maintenance of the dorsal horn interneurons. Proc Natl Acad Sci USA. 2007;104(34):13696-701.

11. Wise EA, Riley JL III, Robinson ME. Clinical pain perception and hormone replacement therapy in postmenopausal women experiencing orofacial pain. Clin J Pain. 2000;16:121-6.

12. Shir Y, Campbell JN, Raja SN, Seltzer Z. The correlation between dietary soy phytoestrogens and neuropathic pain behavior in rats after partial denervation. Anesth Analg. 2002;94:421-6.

13. Ji Y, Murphy AZ, Traub RJ. Estrogen modulation of morphine analgesia of visceral pain in female rats is supraspinally and peripherally mediated. J Pain. 2007;8(6):494-502.

Trabajo recibido el 10 de enero 2011 y aceptado para su publicación el 15 de febrero 2011.

Correspondencia:

Dra. Daisy Flores Cortez

Departamento de Farmacología,

Facultad de Medicina, UNMSM.

Av. Grau 755. Lima 1, Perú.

dafloresc@yahoo.es 